\title{
REVISIÓN
}

\section{Polución aérea y estado protrombótico}

\section{Air pollution and prothrombotic state}

\author{
Jorge Campos ${ }^{1}$, Iván Palomo² \\ ${ }^{1}$ Tecnólogo médico, Magíster(c). Programa de Magíster en Ciencias Biomédicas. Facultad de Ciencias de la Salud, Universidad de Talca, Chile. \\ ${ }^{2}$ Tecnólogo médico, Doctor. Programa de Investigación en Factores de Riesgo de Enfermedad Cardiovascular (PIFRECV), Depto. de Bioquímica Clínica e Inmunohematología, \\ Facultad de Ciencias de la Salud, Universidad de Talca, Chile.
}

\begin{abstract}
Resumen
La polución aérea es una mezcla heterogénea de gases, líquidos y material particulado (MP) presente en el mundo urbano moderno. El MP varía en tamaño, desde pocos nanómetros hasta 10 micras de diámetro. Se ha observado que la exposición a MP se asocia a un mayor riesgo de enfermedades cardiovasculares. Al respecto se ha planteado tres posibles mecanismos: inducción de inflamación sistémica y/o estrés oxidativo; alteraciones en el balance de autonomía cardiaca; y, acción directa sobre el sistema circulatorio, causando vasoconstricción aguda, disfunción endotelial y estado protrombótico. Hay evidencias que indican que la polución aérea se asocia con el acortamiento del tiempo de protrombina, disminución del activador del plasminógeno tisular, aumento de fibrinógeno, factor VIII y factor von Willebrand e hiperactividad plaquetaria.
\end{abstract}

Palabras clave: Polución del aire; agentes de coagulación; plaquetas; protrombina; enfermedades cardiovasculares.

\begin{abstract}
Air pollution is a heterogenic mixture of gases, liquids and particulate matter (PM) present in the urban modern world. PM rates vary in size from a few nanometers to 10 micra diameter. The exposure to PM is associated to a major risk of cardiovascular diseases as explained by three mechanisms: induction of systemic inflammation and/or oxidative stress, alteration in the cardiac autonomic balance and direct action on the circulatory system, causing acute vasoconstriction, endothelial dysfunction and prothrombotic state. There is evidence suggesting that air pollution is associated with shortened prothrombine time, decrease in tissue plasminogen activator, increase of fibrinogen, factor VIII and factor von Willebrand, and platelet hyperactivity.
\end{abstract}

Key words: Air pollution; coagulation agents; blood platelets; prothrombin; cardiovascular diseases.

\section{INTRODUCCIÓN}

La polución aérea se encuentra presente en muchas ciudades en el mundo, tanto en países desarrollados como en países en vías de desarrollo ${ }^{(1-3)}$. Existe evidencia que la polución aérea, caracterizada por un aumento de material particulado (MP) en el aire, incrementa el riesgo a desarrollar enfermedades cardiovasculares $(\mathrm{ECV})^{(1,4-6)}$.

La presente revisión focaliza su atención en los mecanismos asociados a la polución aérea que favorecen el desarrollo de un estado protrombótico.

\section{MATERIAL PARTICULADO}

El MP se origina en combustibles fósiles, como por ejemplo hollín de diesel y adicionalmente otras partículas creadas por transformaciones físico-químicas de gases, tales como formación de nitratos y sulfatos a partir de gases de ácido nítrico y dióxido de sulfuro, respectivamente ${ }^{(1)}$. Entre las numerosas fuentes de MP se incluye: vehículos con motor a emisión, fragmentación de neumáticos y resuspensión de polvo del suelo, generación de energía y otras combustiones industriales, procesamientos de metales, agricultura, demoliciones, construcción, quema casera de leña, pólenes, incendios forestales y roces agrícolas, emisiones volcánicas, aerosoles marítimos, entre otros ${ }^{(1)}$.

Se clasifica el MP según su diámetro, en $\mathrm{MP}_{10}(10 \mu \mathrm{m}), \mathrm{MP}$ grueso $(10$ a $2,5 \mu \mathrm{m}) \mathrm{y}$ MP ultrafino $(<2,5 \mu \mathrm{m})^{(1)}$. Por otro lado, el desarrollo de la nanotecnología y de la ingeniería de nanomateriales del carbón ha permitido generar nanopartículas de 1 a $100 \mathrm{~nm}{ }^{(7)}$, nanotubos de pared simple, nanotubos de pared múltiple y MP estándar de $1,4 \mathrm{~nm}{ }^{(8)}$. También, se ha preparado mezclas de partículas derivadas del carbón, partículas de carbón purificadas poliestireno de $60 \mathrm{~nm}{ }^{(9)}$. Esto implica la interacción directa de material ultrafino en sangre ${ }^{(9,10)}$. Se ha observado que aerosoles con MP entre 7 y $487 \mathrm{~nm}$ de diámetro, marcados $\operatorname{con} \mathrm{Tc}^{99}$, difunden a la circulación ${ }^{(11)}$.
Se ha encontrado que la reducción de la concentración del MP ultrafino en 10 $\mu \mathrm{g} / \mathrm{m}^{3}$ de aire se asocia con un incremento de 0,77 años en la expectativa de vida ${ }^{(12)}$. Por otra parte, se ha observado que la polución aérea se asocia con cambios en la función hemostática global, favoreciendo una tendencia a la hipercoagulabilidad. En ese contexto, se ha descrito que la exposición a altas concentraciones de $\mathrm{MP}_{10}$ durante 30 días acorta el tiempo de protrombina ${ }^{(1)}$.

En Chile, la minería es responsable de parte importante de la polución aérea, existiendo ciudades que han sido declaradas zonas saturadas, por la presencia de alta concentración de $\mathrm{MP}_{10}$ en el aire; además, de vanadio y níquel, es responsable de dicha contaminación la utilización de petcoke, combustible derivado del petróleo, en dos plantas termoeléctricas y relaves mineros abandonados en la ribera del río Copiapó, lo que ha tenido como consecuencia que las enfermedades respiratorias y cardiovasculares registradas en Tocopilla duplicaran a las de Iquique ${ }^{(13)}$. 


\section{HEMOSTASIA: Aspectos Generales}

Una breve descripción de los aspectos fundamentales de la hemostasia facilitará la comprensión de los efectos protrombóticos del MP. A este complejo proceso, clásicamente se le separa en hemostasia primaria, coagulación y fibrinólisis.

Hemostasia primaria. Esta fase incluye la vasoconstricción y las interacciones plaqueta-endotelio y plaqueta-plaqueta. Estas últimas tienen un tamaño de 1,5 a 3 $\mu \mathrm{m}$ y en reposo son de forma discoide. Se originan por fragmentación del citoplasma de los megacariocitos en la médula ósea; pasan a la circulación, donde permanecen por aproximadamente 8 días, tiempo después del cual son retiradas por los macrófagos, principalmente espléni$\cos { }^{(14)}$.

Desde el punto de vista funcional, las plaquetas desarrollan fenómenos de adhesión, secreción y agregación. Las plaquetas se adhieren a la matriz subendotelial a través de uniones entre sus glicoproteínas de membrana (GPIa-IIa, GPIV, GPVI y GPIb-IX-V) y proteínas adhesivas del subendotelio, especialmente colágeno y factor von Willebrand (FVW) ${ }^{(15)}$. Las plaquetas adheridas, al activarse, cambian de forma, desde discoides a esféricas con prolongaciones y presentan cambios conformacionales en el complejo GPIIb-IIIa, lo que permite su unión a fibrinógeno y FVW. Dichas plaquetas además liberan el contenido de sus gránulos alfa (FVW, P-selectina, factor plaquetario 4 y varios factores de la coagulación) y gránulos densos (ADP, ATP y calcio, entre otras moléculas). Además, las plaquetas activadas sintetizan y secretan tromboxano $\mathrm{A}_{2}\left(\mathrm{TXA}_{2}\right)$, molécula que actúa como agonista plaquetario y vasoconstrictor. Por otra parte, las plaquetas activadas favorecen la coagulación, al exponer fosfolípidos aniónicos y expresar factor tisular (FT) ${ }^{(14,15)}$. La hemostasia primaria es regulada principalmente por el óxido nítrico $(\mathrm{NO})$ y la prostaciclina $\left(\mathrm{PGI}_{2}\right)$, moléculas liberadas por el endotelio y que actúan como antiagregantes y vasodilatadoras ${ }^{(10)}$.

Coagulación. El sistema de la coagulación corresponde a una cascada de activación proteolítica de factores plasmáticos ${ }^{(15)}$. In vivo, la coagulación se activa por exposición del FT, el que forma complejo con el FVII. Este último activado, actúa sobre el FX, que con el FV forma el complejo protrombinasa que transforma la protrombina (FII) en trombina, una potente serinoproteasa que actúa sobre el fibrinógeno y lo transforma en fibrina. Ésta al depositarse sobre y entre las plaquetas, estabiliza el tapón plaquetario. La trombina, además de la función antes descrita, cumple otras funciones: agonista plaquetario y activador de varios factores de la coagulación (FVIII, FV, FXI y FXIII), de la proteína C y del TAFI (thrombin activable fibrinolysis inhibitor), un inhibidor de la fibrinólisis. La coagulación está regulada por anticoagulantes naturales, siendo los más importantes la antitrombina III (ATIII), el sistema de la proteína $\mathrm{C}$ y el inhibidor del $\mathrm{FT}^{(16)}$.

Fibrinólisis. Este proceso tiene como objetivo la rotura de los coágulos de fibrina para recanalizar el vaso sanguíneo. El sistema fibrinolítico está compuesto por una serie de activadores e inhibidores ${ }^{(15)}$. El plasminógeno circulante es transformado en plasmina por dos tipos de activadores del plasminógeno (PA), de tipo tisular ( $\mathrm{t}-\mathrm{PA}$ ) y de tipo uroquinasa (u-PA). La plasmina produce la lisis de la fibrina, generándose productos de degradación de la fibrina, entre ellos el dímero D. La fibrinólisis es inhibida por acción de los inhibidores de los PA (PAI-1 y PAI-2), la $\alpha 2$ anti-plasmina y el TAFI ${ }^{(17)}$

\section{POLUCIÓN AÉREA Y ESTADO PRO- TROMBÓTICO}

Existen antecedentes que muestran relación entre la polución aérea y las ECV, pero los mecanismos que subyacen al incremento del riesgo cardiovascular no se les conoce suficientemente ${ }^{(18)}$. Se ha observado que la exposición prolongada a MP favorece el desarrollo de aterosclerosis ${ }^{(10,19)}$. Por otra parte, una vez que el MP llega a los pulmones genera especies reactivas del oxígeno $(\mathrm{ERO})^{(10,20)}$, activando así señales proinflamatorias y causando injuria celular, todo lo cual podría producir los siguientes efectos: (a) reducción de la autonomía cardiaca; (b) paso de MP ultrafino al torrente circulatorio ${ }^{(2,21,22)}$; (c) aumento de los niveles de proteína
C reactiva (PCR) ${ }^{(5,14)}$, interleuquina-6 (IL-6) ${ }^{(23,24)}$, IL-8 ${ }^{(25)}$ y factor de necrosis tumoral (TNF); (d) hipercoagulabilidad (18) (Figura 1). Las citoquinas proinflamatorias causan disfunción endotelial, con el consiguiente deterioro en el flujo coronario y aumento de la presión sanguínea. Los efectos antes mencionados provocan alteraciones en el ritmo y contractibilidad cardiaca e inflamación ${ }^{(20)}$.

Material particulado y endotelio. La polución aérea afecta la función vascular ${ }^{(5,9,18)}$. $\mathrm{MP}_{10}$ altera el endotelio, interactuando directamente con las células endoteliales y su relación con la expresión de genes relacionados con trombogénesis y fibrinólisis, causando un daño agudo en la producción de t-PA, produciendo un desequilibrio entre los factores profibrinolíticos y sus inhibidores endógenos, impidiendo de este modo la disolución del trombo ${ }^{(10)}$.

Material particulado y coagulación. Se ha comunicado hipercoagulabilidad asociada a la polución del aire ${ }^{(1)}$. El MP ultrafino y nanopartículas estimulan la síntesis de FT en endotelio y macrófagos ${ }^{(21,25,26)}$. El tiempo de protrombina (TP) se ve acortado en individuos expuestos por 30 días en ambientes con altas concentraciones de $\mathrm{MP}_{10}{ }^{(1)}$. Por otro lado, esta misma condición no altera el tiempo de tromboplastina parcial activado (TTPA) (9,26) , los niveles de ATIII o de proteína C (1). La polución aérea también aumenta la viscosidad plasmática, lo que ha sido correlacionado positivamente con los niveles plasmáticos de fibrinógeno, que aumentan en presencia de MP ${ }^{(21)}$. Por otra parte, la exposición al humo de madera con altas concentraciones de MP incrementa la actividad de FVIII en el plasma ${ }^{(27)}$.

Material particulado y fibrinólisis. La inhibición de la fibrinólisis favorece un estado protrombótico. El MP reduce los niveles de t-PA y del PAI-1 ${ }^{(10)}$. Alteraciones en el balance entre t-PA y PAI-1 a nivel pulmonar han sido asociadas con estados inflamatorios, tales como neumonía, sepsis, distrés respiratorio del adulto y estado postoperatorio. Se ha observado que las células endoteliales en presencia de $\mathrm{MP}_{10}$ aumentan su actividad procoagulante y disminuyen su capacidad fibrinolítica ${ }^{(25)}$ 


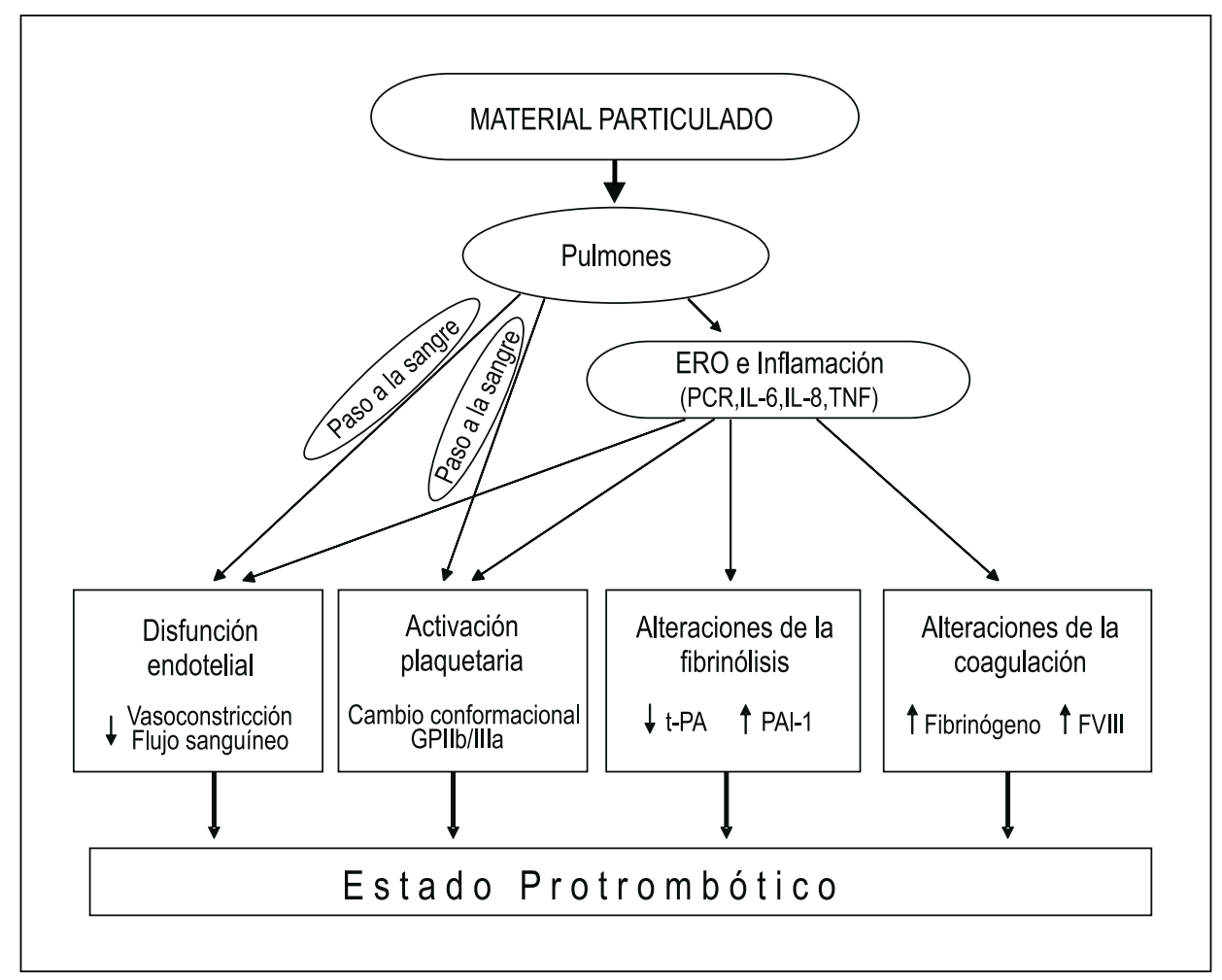

Figura 1. Fisiopatología del efecto protrombótico del material particulado. ERO, Especies reactivas del oxigeno; PCR, Proteína C reactiva; IL-6, Interleuquina-6; IL-8, Interleuquina-8; TNF, Factor de necrosis tumoral; t-PA, Activador del plasminógeno tisular; PAI-1, Inhibidor del activador del plasminógeno tisular-1; FVIII, Factor VIII.

Material particulado y plaquetas. Se ha descrito que nanopartículas de carbón inducen agregación plaquetaria en forma dependiente de la concentración ${ }^{(7)}$. Entre los mecanismos que explicarían la activación plaquetaria, se ha sugerido que ocurrirían cambios conformacionales en el complejo GPIIb/IIIa y mayor exposición de P-selectina en la membrana plaquetaria, lo que favorece la interacción plaqueta-leucocito ${ }^{(6)}$. Por otro lado, se ha observado que nanopartículas inducen la liberación de $\mathrm{TXA}_{2}$ y ADP, y la activación de la vía proteína kinasa $\mathrm{C}$ en las plaquetas; además, libera metaloproteinasas desde la matriz endotelial. Por otra parte, las nanopartículas de carbón favorecen el desarrollo de trombosis en ratas ${ }^{(9)}$. También, se ha descrito que la agregación plaquetaria inducida por MP es inhibida por $\mathrm{PGI}_{2}$, lo que no ocurriría con aspirina ${ }^{(7)}$. Además, existe evidencia que los nanotubos forman puentes moleculares entre plaquetas ${ }^{(7)}$; las propiedades eléctricas del MP y de los nanotubos podrían participar en la interacción de estas estructuras con el complejo GPIIb/ IIIa, induciendo la activación plaquetaria ${ }^{(8,9)}$. Por otro lado, la presencia de metales de transición, como el hierro y el cobre, que se caracterizan por presentar diversos estados de oxidación y poseer una fuerte energía catalítica, son capaces de generar ERO implicadas en peroxidación lipídica y causar daño endotelial y activación plaquetaria $^{(28)}$. Asimismo, MP ultrafino, constituido especialmente por aminas de poliestireno cargado positivamente, interactúa con las cargas negativas de la superficie plaquetaria, dadas principalmente por ácido siálico, forma puentes entre las plaquetas, favoreciendo la agregación plaquetaria. Dicho efecto fue observado en ratas dentro de la primera hora de instilación intratraqueal de MP ultrafino, pudiendo ser resultado del pasaje de este a la circulación ${ }^{(9)}$. Otro mecanismo que podría inducir la agregación plaquetaria es la bioinmovilización de proteínas, propiedad que presentan los nanotubos; grupos aminos de proteínas de la membrana plaquetaria podrían ser sustituidos nucleofílicamente por grupos $\mathrm{N}$-hidroxisuccinimida aportados por la pared de los nanotubos, resultando en la formación de enlaces no covalentes de amida $^{(8)}$.

Concluimos de la presente revisión que el MP y las nanopartículas pueden presentar efectos tóxico-biológicos como inflamación, estrés oxidativo, desbalance de la autonomía cardiaca y estado protrombótico, con subsecuentes efectos para la salud cardiovascular. El MP y las nanopartículas causan alteración endotelial, aumento de algunos factores de la coagulación, alteración de la fibrinólisis y activación plaquetaria, todo lo cual favorece el desarrollo de un estado protrombótico.

\section{REFERENCIAS BIBLIOGRÁFICAS}

1. Lippi G, Favaloro EJ, Franchini M, Guidi GC. Air pollution and coagulation testing: A new source of biological variability? Thrombosis Research. 2008;123(1):50-4. 
2. Division of Cardiovascular Medicine, University of Michigan, Ann Arbor, MI 48106-0739, USA. Clinical Science (London, England: 1979) Cardiovascular effects of air pollution. Clinical Science. 2008;115:175-87.

3. Vichit-Vadakan N, Vajanapoom N, Ostro B. The Public Health and Air Pollution in Asia (PAPA) Project: Estimating the Mortality Effects of Particulate Matter in Bangkok, Thailand. Environmental Health Perspectives. 2008;116:1179-82.

4. Simkhovich BZ, Kleinman MT, Kloner RA. Air pollution and cardiovascular injury epidemiology, toxicology, and mechanisms. J Am Coll Cardiol. 2008;52:719-26.

5. Kaufman D. Air pollution and mortality: are we closer to understanding the how? Am J Respir Crit Care Med. 2007;176:325-6.

6. Ray MR, Mukherjee1 S, Roychoudhury S, Bhattacharya $P$, Banerjee $M$, Siddique $S$, et al. Platelet activation, upregulation of CD11b/CD18 expression on leukocytes and increase in circulating leukocyte-platelet aggregates in Indian women chronically exposed to biomass smoke. Human Experim Toxicol. 2006;25:627-35.

7. Radomski A, Jurasz P, Alonso-Escolano D, Drews M, Morandi M, Malilinski T, et al. Nanoparticle-induced platelet aggregation and vascular thrombosis. $\mathrm{Br}$ J Pharmacol. 2005;146:882-93.

8. DAl H. Carbon nanotubes: synthesis, integration, and properties. Accounts Chem Res. 2002;35:103544.

9. Nemmars A, Hoylaerts M, Hoet P, Dinsdale D, Smith $\mathrm{T}, \mathrm{Xu} \mathrm{H}$, et al. Ultrafine particles affect experimental thrombosis in an in vivo hamster model. Am J Respir Crit Care Med. 2002;166:998-1004.

10. Mills N, Törnqvist H, Robinson S, Gonzalez M, Söderberg S, Sandström T, et al. Air pollution and atherothrombosis. Inhalation Toxicol. 2007;19:819.

11. Mills N, Amin N, Robinson S, Anand A, Davies J, Patel D, et al. Do inhaled carbon nanoparticles translocate directly into the circulation in humans?
Am J Respir Crit Care Med. 2006;173:426-31.

12. Pope A, Ezzati M, Dockery D. Fine-particulate air pollution and life expectancy in the United States. New Engl J Med. 2009;360(4):376-86.

13. Informe anual sobre derechos humanos en Chile: hechos de 2006. Santiago, Chile: Universidad Diego Portales; 2007.

14. Santos MT, Aranda E, Valles J, Palomo I. Hemostasia primaria. En: Palomo I, Pereira J, Palma J. Hematología: Fisiopatología y Diagnóstico. Cap. 19. Talca, Chile: Editorial Universidad de Talca; 2005.

15. Palomo I, Pereira J, Palma J. Hematología: Fisiopatología y Diagnóstico. Cap. 20. Talca, Chile: Editorial Universidad de Talca; 2005.

16. Feistritzer C, Wiedermann CJ. Effects of anticoagulant strategies on activation of inflammation and coagulation. Expert Opinion Biology Ther. 2007;7:855-70.

17. Marx PF, Brondijk TH, Plug T, Romijn RA, Hemrika W, Meijers JC, et al. Crystal structures of TAFI elucidate the inactivation mechanism of activated TAFI: a novel mechanism for enzyme autoregulation. Blood. 2008;112:2597-608.

18. Baccarelli A, Zanobetti A, Martinelli I, Grillo P, Hou L, Giacomini S, et al. Effects of exposure to air pollution on blood coagulation. J Thrombosis Haemostasis. 2007;5:252-60.

19. Gong KW, Zhao W, Li N, Barajas B, Kleinman $M$, Sioutas $C$, et al. Air-pollutant chemicals and oxidized lipids exhibit genome-wide synergistic effects on endothelial cells. Genome Biol. 2007;8: R149.

20.Zwaal RF, Comfurius P, Bevers EM. Lipid-protein interactions in blood. Biochim Biophys Acta. 1998;1376:433-53.

21. Nemmar A, Hoylaerts M, Hoet P, Nemery B. Possible mechanisms of the cardiovascular effects of inhaled particles: systemic translocation and protrombotic effects. Toxicology Letters. 2004;149:243-53.

22. Chuang KJ, Chan C, Su T, Lee C, Tang C. The effect of urban air pollution on inflammation, oxidative stress, coagulation, and autonomic dysfunction in young adults. Am J Respir Crit Care Med.2007; 176:370-6.

23. Rückerl R, Greven S, Ljungman P, Aalto $P$, Antoniades C, Bellander T, et al. Air pollution and inflammation (interleukin-6, C-reactive protein, fibrinogen) in myocardial infarction survivors. Environ Health Perspect. 2007;115:1072-80.

24. White J, Platelet structure. En: Michelson AD (Editor). Platelets. Cap. 3. San Diego: Academic Press; 2007.

25. Seaton A, Crawford V, Elton R, Mcnerlan S, Cherrie J, Watt $M$, et al. Particulate air pollution and the blood. Thorax. 1999;54:1027-32.

26. Vermylen J, Hoylaerts MF. The procoagulant effects of air pollution. J Thromb Haemost. 2007;5:2501.

27. Barregard L, Sällsten G, Gustafson P, Andersson L, Johansson L, Basu S, et al. Experimental exposure to wood-smoke particles in healthy humans: effects on markers of inflammation, coagulation, and lipid peroxidation. Inhal Toxicol. 2006;18:84553.

28. Widlansky M, Gokce N, Keaney J, Vita J. The clinical implications of endothelial dysfunction. J Am Coll Cardiol. 2003;42:1149-60.

Manuscrito recibido el 27 de febrero de 2009 y aceptado para publicación el 30 de marzo de 2009.

Correspondencia:

Iván Palomo G.

Departamento de Bioquímica Clínica e Inmunohematología, Facultad Ciencias de la Salud, Universidad de Talca, Talca, Chile P.0. box: 747, Talca, Chile

Correo-e: ipalomo@utalca.cl 